\title{
Two-phonon scattering of magnetorotons in fractional quantum Hall liquids
}

\author{
V. M. Apalkov* and M. E. Portnoi ${ }^{\dagger}$ \\ School of Physics, University of Exeter, Stocker Road, Exeter EX4 4QL, United Kingdom
}

(Received 26 June 2002; published 10 September 2002)

\begin{abstract}
We study the phonon-assisted process of dissociation of a magnetoroton, in a fractional quantum Hall liquid, into an unbound pair of quasiparticles. Whilst the dissociation is forbidden to first order in the electron-phonon interaction, it can occur as a two-phonon process. Depending on the value of final separation between the quasiparticles, the dissociation is either a single event involving the absorption of one phonon and the emission of another phonon of similar energy, or a two-phonon diffusion of a quasiexciton in momentum space. The dependence of the magnetoroton dissociation time on the filling factor of the incompressible liquid is found.
\end{abstract}

DOI: 10.1103/PhysRevB.66.121303

PACS number(s): 73.43.-f, 73.43.Fj

Recent advances in phonon spectroscopy ${ }^{1}$ have yielded fascinating results in several branches of condensed-matter physics. These results range from the observation of the remarkable anisotropy of phonon propagation in crystalline solids ${ }^{2}$ to the detection of $R^{-}$rotons in liquid helium. ${ }^{3} \mathrm{Ar}-$ guably the most interesting results are obtained when ballistic nonequilibrium phonons interact with low-dimensional many-body systems. For example, recent experiments in phonon-induced quantum evaporation have provided the first direct evidence for a Bose-Einstein condensate on the surface of liquid ${ }^{4} \mathrm{He}^{4} \mathrm{~A}$ similar approach has been used to probe quasi-two-dimensional (2D) electron systems based on semiconductor heterostructures. In this method, the nonequilibrium acoustic phonons injected into the system are absorbed by the $2 \mathrm{D}$ electron gas, and this changes its transport ${ }^{5-8}$ and optical $^{9}$ properties at low temperatures. Such a technique has recently been used to obtain information about a magnetically quantized 2D electron gas at fractional filling factors. ${ }^{7}$

The absorption of nonequilibrium phonons by a 2D incompressible liquid, underlying the fractional quantum Hall effect, results in an increase of the effective electron temperature, which is detected by measuring the dissipative conductivity of the system. The phonon absorption process can be schematically divided into two stages. During the first stage, the phonons are absorbed by the incompressible liquid, with the creation of nonequilibrium neutral excitations (magnetorotons) with wave vectors of the order of the inverse magnetic length. ${ }^{10}$ During the second stage, the nonequilibrium electron system is thermalized, with the creation of charged quasiparticles which are free to participate in the dissipative conductivity. The process of thermalization can be understood as the dissociation of magnetorotons into well-separated quasielectrons and quasiholes. Initially, it was proposed that such a process could be intensified by the absorption of nonequilibrium phonons from the pulse. ${ }^{11}$ It was later shown ${ }^{12}$ that due to the almost flat dispersion of the neutral excitations of an incompressible liquid at large wave vectors, the absorption of a phonon by a magnetoroton is allowed only for a very small momentum transfer, which is not enough to dissociate the magnetoroton. However, due to the electroneutrality of the magnetoroton, the detected changes in dissipative conductivity cannot be explained without the dissociation of the magnetoroton into charged quasiparticles. The main goal of this paper is to uncover the mechanism of magnetoroton dissociation. Understanding this mechanism is essential for the interpretation of the phonon absorption spectroscopy data, and for the prediction of the possible outcome of experiments at different filling factors. We show that the phonon-pulse-induced dissociation of the magnetoroton occurs as a second-order process in the electron-phonon interaction. It is a common feature for all systems containing dispersionless particles that two-phonon processes provide the main contribution to the transport properties of the system. ${ }^{13,14}$

The neutral low-energy excitations of the incompressible electron liquid have a nonzero gap $\Delta(q)$ for all wave vectors $q$, with a minimum value $\Delta^{*}$ at $q=q^{*} \sim 1 / l_{0}$, where $l_{0}$ is the magnetic length. The excitations close to $q^{*}$ are well described by the single-mode approximation, ${ }^{15}$ and are called magnetorotons by analogy with roton excitations of superfluid helium. At large wave vectors the neutral excitation is a quasiexciton, which consists of a fractionally-charged quasielectron and quasihole. ${ }^{15-18}$ In what follows, we consider quantum Hall liquids with a filling factor $\nu=p / m$, where $p$ is an integer and $m$ is an odd number. For this filling factor the charge of a quasielectron (quasihole) is $1 / m(-1 / m)$ of the electron charge. The effective magnetic length for these quasiparticles is $l_{0}^{*}=\sqrt{m} l_{0}$. The dispersion of neutral excitations of the incompressible liquid at large values of momentum can then be written in the form $\Delta(q)=\Delta_{\infty}-\varepsilon_{0} / m^{3} q$, where $\varepsilon_{0}=e^{2} / \kappa l_{0}$ is the Coulomb energy. We use the magnetic length $l_{0}$ and the Coulomb energy $\varepsilon_{0}$ as the units of length and energy, respectively. To study the phonon-induced dissociation of a magnetoroton, we should consider phononassisted transitions of the magnetoroton with momentum close to $q^{*}$ into a quasiexciton with momentum larger than a critical momentum value $q_{c r}$. This critical momentum is defined by the condition

$$
\Delta_{\infty}-\Delta\left(q_{c r}\right)=T_{c r},
$$

where $T_{c r}$ is a characteristic temperature (in units of $\varepsilon_{0} / k_{B}$ ).

To find the two-phonon scattering rate for neutral excitations of an incompressible liquid, we assume that within the whole range of our interest $\left(q>q^{*}\right)$, a magnetoroton can be described as a quasiexciton, i.e., a bound state of a quasielectron and a quasihole. Although this is not a very good approximation at $q \sim q^{*}$, we use this assumption to illustrate 
the importance of two-phonon processes for the thermal relaxation of incompressible liquids.

For convenience we label the $3 \mathrm{D}$ vectors with capital letters, e.g., $\vec{Q}$, and their projections with the corresponding lowercase letters: $\vec{Q}=\left(\vec{q}, q_{z}\right)$. We neglect the effects related to phonon polarization and crystal anisotropy, and write the quasielectron-phonon and quasihole-phonon interaction Hamiltonian in the form

$$
\begin{aligned}
H_{i n t}= & \sum_{\vec{Q}} \frac{1}{m} \frac{V_{e}(Q)}{\sqrt{\Omega}} Z\left(q_{z}\right) \hat{\rho}_{e}(\vec{q})\left[\hat{b}^{\dagger}(-\vec{Q})+\hat{b}(\vec{Q})\right] \\
& -\sum_{\vec{Q}} \frac{1}{m} \frac{V_{e}(Q)}{\sqrt{\Omega}} Z\left(q_{z}\right) \hat{\rho}_{h}(\vec{q})\left[\hat{b}^{\dagger}(-\vec{Q})+\hat{b}(\vec{Q})\right],
\end{aligned}
$$

where $\hat{b}^{\dagger}(\vec{Q})$ and $\hat{b}(\vec{Q})$ are creation and annihilation operators of a phonon with momentum $\vec{Q}, \Omega$ is a normalization volume, and $\hat{\rho}_{e}$ and $\hat{\rho}_{h}$ are the quasielectron and quasihole 2D density operators, respectively. Because the quasiparticles are in the lowest subband of size quantization, the $z$ part of the density operator is accumulated in the form factor $Z\left(q_{z}\right)$ :

$$
Z\left(q_{z}\right)=\frac{1}{\left(1+i q_{z} / \beta\right)^{3}},
$$

where the Fang-Howard approximation ${ }^{19}$ for the lowest subband wave function is used, and $\beta$ is the parameter of the Fang-Howard wave function.

The response of a quasiparticle to the external perturbation is $m$ times weaker than the corresponding response of an electron. For the quasihole this response has the opposite sign. Therefore, in Eq. (2) the quasiparticle-phonon interaction is characterized by the matrix elements $\pm(1 / m) V_{e}(Q)$, where the upper sign is for quasielectrons and the lower sign is for quasiholes. Here, $V_{e}(Q)$ is the matrix element of the electron-phonon interaction, which is given by the expression $^{20}$

$$
V_{e}(Q)=\sqrt{\frac{B_{p}}{Q}}-i \sqrt{B_{d} Q}
$$

where $B_{p}$ and $B_{q}$ are the constants of piezoelectric and deformation potential couplings. We use material parameters of $\mathrm{GaAs}$ in our numerical estimates. form

Using Eq. (4), one can rewrite the Hamiltonian (2) in the

$$
\begin{aligned}
H_{i n t}= & \sum_{\vec{Q}} \frac{1}{m} \frac{Z\left(q_{z}\right)}{\sqrt{\Omega}}\left(\sqrt{\frac{B_{p}}{Q}}-i \sqrt{B_{d} Q}\right) \hat{c}(\vec{q})\left[\hat{b}^{\dagger}(-\vec{Q})\right. \\
& +\hat{b}(\vec{Q})],
\end{aligned}
$$

where $\hat{c}(\vec{q})=\hat{\rho}_{e}(\vec{q})-\hat{\rho}_{h}(\vec{q})$ is the quasiparticle chargedensity operator.

The matrix elements of the operator $\hat{c}(\vec{q})$ between the quasiexciton states, which are characterized by the $2 \mathrm{D}$ momenta $\vec{k}$ and $\vec{k}_{1}$, are given by the expression

$$
\left\langle\vec{k}_{1}|\hat{c}(\vec{q})| \vec{k}\right\rangle=-2 i e^{-m q^{2} / 4} \sin \left(m \frac{q_{x} k_{y}-q_{y} k_{x}}{2}\right) \delta\left(\vec{k}_{1}-\vec{k}-\vec{q}\right),
$$

where the momentum is in units of $1 / l_{0}$.

The rate of the two-phonon scattering of the quasiexciton to states with momentum greater than $q_{c r}$ is

$$
\begin{aligned}
W\left(q_{c r}\right)= & \frac{2 \pi}{\hbar \varepsilon_{0}^{3} l_{0}^{6}} \frac{1}{m^{4}} \int \frac{d \varphi_{q^{*}}}{2 \pi} \int_{q_{f}>q_{c r}} d^{2} \vec{q}_{f} \iint \frac{d^{3} \vec{Q}}{(2 \pi)^{3}} \frac{d^{3} \vec{Q}^{\prime}}{(2 \pi)^{3}}\left|Z\left(q_{z}\right)\right|^{2}\left|Z\left(q_{z}^{\prime}\right)\right|^{2}\left|M\left(\vec{q}^{*}, \vec{q}_{f}, \vec{Q}, \vec{Q}^{\prime}\right)\right|^{2} \\
& \times N(Q)\left[N\left(Q^{\prime}\right)+1\right] \delta\left(\Delta\left(q^{*}\right)-\Delta\left(q_{f}\right)-s\left(Q^{\prime}-Q\right)\right) \delta\left(\vec{q}^{*}+\vec{q}-\vec{q}_{f}-\vec{q}^{\prime}\right),
\end{aligned}
$$

where

$$
\begin{gathered}
M\left(\vec{q}^{*}, \vec{q}_{f}, \vec{Q}, \vec{Q}^{\prime}\right)=\frac{\left\langle\vec{q}^{*}|\hat{H}(\vec{Q})| \vec{q}^{*}+\vec{q}\right\rangle\left\langle\vec{q}^{*}+\vec{q}\left|\hat{H}\left(\vec{Q}^{\prime}\right)\right| \vec{q}_{f}\right\rangle}{\Delta_{a}-s Q^{\prime}}+\frac{\left\langle\vec{q}^{*}\left|\hat{H}\left(\vec{Q}^{\prime}\right)\right| \vec{q}^{*}+\vec{q}^{\prime}\right\rangle\left\langle\vec{q}^{*}+\vec{q}^{\prime}|\hat{H}(\vec{Q})| \vec{q}_{f}\right\rangle}{\Delta_{b}+s Q^{\prime}}, \\
\Delta_{a}=\Delta\left(\vec{q}^{*}+\vec{q}\right)-\Delta\left(\vec{q}_{f}\right), \Delta_{b}=\Delta\left(\vec{q}^{*}+\vec{q}^{\prime}\right)-\Delta\left(\vec{q}^{*}\right), \text { and } \\
\hat{H}(\vec{Q})=\left[\sqrt{\frac{B_{p} l_{0}}{Q}}-i \sqrt{\frac{B_{d} Q}{l_{0}}}\right] \hat{c}(\vec{q}),
\end{gathered}
$$

where $s$ is the average speed of sound, which is $s \approx 0.03$ in units of $\varepsilon_{0} l_{0} / \hbar$. In Eq. (7), the first integral is the average of the scattering rate over all possible directions of the initial momentum $\vec{q}^{*} . N(Q)$ is the nonequilibrium phonon distribution function created by the external phonon source. The two $\delta$-functions represent the conservation of energy and momentum.

Due to the exponential dependence [Eq. (6)] of the matrix elements of the charge-density operator $\hat{c}(\vec{q})$ on the phonon momentum $q$, the main contribution to the integrals in Eq. (7) comes from the region where the difference between $q$ and $q^{\prime}$ is small, and the vectors $\vec{q}^{*}$ and $\vec{q}_{f}$ are parallel. In this case, substituting Eqs. (6) and (9) into Eq. (8), we obtain the expression 


$$
\begin{aligned}
\left|M\left(\vec{q}^{*}, \vec{q}_{f}, \vec{Q}, \vec{Q}^{\prime}\right)\right|^{2} \approx & 16 \frac{\left(B_{p} l_{0}^{2}+B_{d} Q^{2}\right)\left(B_{p} l_{0}^{2}+B_{d} Q^{\prime} \xi\right.}{Q Q^{\prime} l_{0}^{2}\left[\Delta_{1}\left(q_{f}\right)+s Q^{\prime}\right]^{2}}\left\{\left[\frac{\delta \Delta}{\Delta_{1}\left(q_{f}\right)+s Q^{\prime}}\right]^{2}\left[\sin \frac{m q q^{*} \sin \phi}{2}\right]^{4}\right. \\
& \left.\times\left[\cos \frac{m q q^{\prime} \sin 2 \phi}{2}\right]^{2}+\left[\sin \frac{m q q^{\prime} \sin 2 \phi}{2} \sin \frac{m q q_{f} \sin 2 \psi}{2}\right]^{2}\right\},
\end{aligned}
$$

where $\Delta_{1}\left(q_{c r}\right) \approx \Delta_{a} \approx-\Delta_{b} \approx \Delta\left(\left[q_{c r}+q^{*}\right] / 2\right)-\Delta\left(q^{*}\right), \quad \delta \Delta$ $=\Delta_{a}+\Delta_{b} \approx 2 \Delta\left(\vec{q}^{*}+\vec{q}\right)-\Delta\left(\vec{q}_{f}\right)-\Delta\left(\vec{q}^{*}\right), \phi$ is the angle between vectors $\vec{q}$ and $\vec{q}^{*}$, and $\psi$ is the angle between vectors $\vec{q}^{*}$ and $\vec{q}_{f}$. From Eq. (10), one can see that the amplitude of two-phonon scattering of the quasiexciton vanishes when the wave vectors of the phonons, $\vec{q}$ and $\vec{q}^{\prime}$, have the same direction. We assume that the angles $\phi$ and $\psi$ between the wave vectors are small. This approximation is good for large values of $q_{f}$. The first term in the curly brackets in Eq. (10) contains the factor $\delta \Delta$, which describes the nonlinearity of the excitation spectra and depends strongly on the shape of the dispersion curve $\Delta(q)$. There is no good approximation to describe the magnetoroton spectrum in the intermediate region between the magnetoroton minimum and the beginning of the quasiexcitonlike dispersion. As an upper limit for $\delta \Delta$ we take the value $\delta \Delta=0.005$, which corresponds to a quarter of the magnetoroton binding energy; the term containing $\delta \Delta$ then gives only a small correction to the whole expression. In what follows, we shall disregard this term and only consider the contribution from the second term in the curly brackets.

If the width of the wave function in the $z$ direction is much larger than $2 / \delta q_{c r}$, i.e., the Fang-Howard parameter $\beta$ is much smaller than $\delta q_{c r} / 2$, then by substituting Eq. (10) into Eq. (7) and performing the integrations, we obtain the expression for the rate of quasiexciton dissociation:

$$
\begin{aligned}
W\left(q_{c r}\right) \approx & W_{0} \frac{1}{m^{4}} \frac{3}{2 \pi^{2}} \frac{1}{s^{3}} \frac{q_{c r} \sqrt{q_{c r}}}{\sqrt{q^{*}}}\left[\frac{\beta^{2} s}{\delta q_{c r} \Delta_{0}\left(q_{c r}\right)}\right]^{7 / 2} \\
& \times N\left(\delta q_{c r} / 2\right)\left[N\left(\delta q_{c r} / 2\right)+1\right] \\
& \times\left[1+\frac{B_{d} \delta q_{c r}^{2}}{4 B_{p} l_{0}^{2}}\right]^{2} \frac{1}{\delta q_{c r}} \\
& \times\left(1+3 \frac{q_{c r}}{\left(q^{*}+q_{c r}\right)^{2}}\right) \operatorname{erfc}\left(\frac{\sqrt{m}}{2} \delta q_{c r}\right)
\end{aligned}
$$

where

$$
\begin{gathered}
\delta q_{c r}=q_{c r}-q^{*} \\
\operatorname{erfc}(x)=1-\operatorname{erf}(x)=(2 / \sqrt{\pi}) \int_{x}^{\infty} d y \exp \left(-y^{2}\right)
\end{gathered}
$$

is the complementary error function, and the constant $W_{0}$ is given by $W_{0}=B_{p}^{2} /\left(\hbar \varepsilon_{0}^{3} l_{0}^{4}\right)$.
In the opposite case of a narrow quantum well, i.e., $\beta$ $\gg \delta q_{c r} / 2, W\left(q_{c r}\right)$ takes the form

$$
\begin{aligned}
W\left(q_{c r}\right) \approx & W_{0} \frac{1}{m^{4}} \frac{1}{6 \pi^{3}} \frac{1}{s^{3}} \frac{q_{c r} \sqrt{q_{c r}}}{\sqrt{q^{*}}} N\left(\delta q_{c r} / 2\right)\left[N\left(\delta q_{c r} / 2\right)+1\right] \\
& \times \frac{1}{\delta q_{c r}}\left(1+3 \frac{q_{c r}}{\left(q^{*}+q_{c r}\right)^{2}}\right) \operatorname{erfc}\left(\frac{\sqrt{m}}{2} \delta q_{c r}\right)
\end{aligned}
$$

where the main contribution comes from the piezoelectric interaction.

From Eqs. (11) and (12) one can see that the rate $W\left(q_{c r}\right)$ is exponentially small for large $q_{c r}$. The two-phonon dissociation of the quasiexciton as a single event is allowed only for small values of critical momentum. For example, for $q_{c r} \approx 3$, which corresponds to a characteristic temperature larger than $T_{c r} \sim 1 / m^{3} q_{c r} \sim 1.5 \mathrm{~K}$ [see Eq. (1)], we find from Eq. (12) the rate of quasiexciton dissociation $W\left(q_{c r}\right) \approx 3$ $\times 10^{8} \mathrm{~s}^{-1}$, or the characteristic time of dissociation $\sim 3$ $\times 10^{-9}$ s. For this calculation the temperature of nonequilibrium phonons is taken as $T_{p h}=10 \mathrm{~K}$. For a larger value of the critical momentum $q_{c r}$, the rate of direct two-phonon dissociation of a quasiexciton becomes very small. For example, at $T_{c r}=0.5 \mathrm{~K}$ we obtain $q_{c r} \sim 1 / m^{3} T_{c r} \sim 7$, which gives an exponentially small value of the erfc function: $\operatorname{erfc}\left[(\sqrt{3} / 2)\left(q_{c r}-q^{*}\right)\right] \sim 10^{-16}$. In this case one should consider the series of subsequent two-phonon transitions, which represent the diffusion of the quasiexciton in momentum space.

Following the derivation of Eqs. (11) and (12), we estimate the average change in the momentum of the quasiexciton, $\delta q_{0}$, during a two-phonon scattering event: $\delta q_{0}$ $\approx 2 / \sqrt{m}$, which is equal to 1.15 for $\nu=1 / 3$.

The time of the two-phonon scattering event can be estimated from Eq. (7), where the lower integration limit is taken as $q^{*}\left(q_{f}>q^{*}\right)$. For a narrow quantum well, this time is given by

$$
\begin{aligned}
\tau_{0} \approx & \frac{1}{W_{0}} 6 \pi^{3} m^{4} \frac{s^{3}}{N\left(\delta q_{0} / 2\right)\left[N\left(\delta q_{0} / 2\right)+1\right]} \\
& \times \frac{\sqrt{q^{*}}}{\left(q^{*}+\delta q_{0}\right)^{3 / 2}} \delta q_{0}\left(1+3 \frac{q_{c r}}{\left(q^{*}+q_{c r}\right)^{2}}\right)^{-1}
\end{aligned}
$$

The time of diffusion of the quasiexciton, from the magnetoroton state with momentum $q^{*}$ to the quasiexciton state with critical momentum $q_{c r}$, can then be estimated from the equation

$$
\tau_{c r} \approx \tau_{0}\left(\frac{q_{c r}}{\delta q_{0}}\right)^{2} \approx \tau_{0} \frac{1}{m^{6}}\left(\frac{1}{T_{c r} \delta q_{0}}\right)^{2}
$$


where Eq. (1) has been used.

For $\nu=1 / 3, s=0.03$, and for the nonequilibrium phonon temperature $T_{p h}=10 \mathrm{~K}$, our estimate gives $\tau_{0} \approx 0.4$ $\times 10^{-10} \mathrm{~s}$. In this case the time of the magnetoroton dissociation is about $\tau_{c r} \approx 2 \times 10^{-9} \mathrm{~s}$ for $T_{c r}=0.5 \mathrm{~K}$.

We assume that the phonon distribution is characterized by some equilibrium temperature $T_{p h}$ of the phonon pulse: $N(q)=\left(e^{s q / T_{p h}}-1\right)^{-1}$. The typical phonon energy in Eq. (13) is about $s \delta q_{0} / 2=s / \sqrt{m}$, which corresponds to a temperature $T_{p h}^{*} \approx s / \sqrt{m}$. At $\nu=1 / 3$ we have $T_{p h}^{*} \approx 2 \mathrm{~K}$. If the phonon nonequilibrium temperature is much larger than $T_{p h}^{*}, T_{p h} \gg T_{p h}^{*}$, then the dependence of $\tau_{0}$ on the denominator $m$ of the filling factor has the power law $\tau_{0} \propto m^{7 / 2}$. From Eq. (14), the time of magnetoroton dissociation $\tau_{c r}$ is then proportional to $m^{-3 / 2}$.

In conclusion, we have shown that a flux of nonequilibrium phonons induces dissociation of magnetorotons of an incompressible fractional quantum Hall liquid into wellseparated pairs of quasiparticles. This process is only possible to second order in the quasiparticle-phonon interaction. Depending on the value of the critical quasiexciton momentum, the magnetoroton decay can be considered as a direct two-phonon dissociation or as the result of the two-phonon diffusion of a quasiexciton in momentum space.

We are grateful to K. A. Benedict, C. J. Mellor, and A. F. G. Wyatt for stimulating discussions and to D. G. W. Parfitt for valuable advice. This work was supported by the UK EPSRC.
*Present address: Department of Physics, University of Utah, Salt Lake City, Utah 84112.

†Electronic address: m.e.portnoi@ex.ac.uk

${ }^{1}$ The state of the art in phonon spectroscopy is best represented in Proceedings of the Tenth International Conference on Phonon Scattering in Condensed Matter, edited by M. N. Wybourne et al. [Physica B 316-317 (2002)].

${ }^{2}$ J.P. Wolfe, Phys. Today 48(9), 34 (1995).

${ }^{3}$ M.A.H. Tucker and A.F.G. Wyatt, Science 283, 1150 (1999).

${ }^{4}$ A.F.G. Wyatt, Nature (London) 391, 56 (1998); see also A. Griffin, ibid. 391, 25 (1998).

${ }^{5}$ L. J. Challis and A. J. Kent, in Aspects of Semiconductor Nanostructures, edited by G. Bauer, F. Kuchar, and H. Heinrich (Springer, Heidelberg, 1992).

${ }^{6}$ C.J. Mellor, R.H. Eyles, J.E. Digby, A.J. Kent, K.A. Benedict, L.J. Challis, M. Henini, C.T. Foxon, and J.J. Harris, Phys. Rev. Lett. 74, 2339 (1995).

${ }^{7}$ U. Zeitler, A.M. Devitt, J.E. Digby, C.J. Mellor, A.J. Kent, K.A. Benedict, and T. Cheng, Phys. Rev. Lett. 82, 5333 (1999).

${ }^{8}$ F. Schultze-Wischler, U. Zeitler, F. Hohls, R.J. Haug, D. Reuter, and A.D. Wieck, Physica E (Amsterdam) 12, 474 (2002).

${ }^{9}$ V.E. Zhitomirskii, O.V. Volkov, I.V. Kukushkin, S. Rozhko, and W. Dietsche, Pis'ma Zh. Eksp. Teor. Fiz. 69, 30 (1999) [JETP
Lett. 69, 31 (1999)]

${ }^{10}$ K.A. Benedict, R.K. Hills, and C.J. Mellor, Phys. Rev. B 60, 10 984 (1999).

${ }^{11}$ K. A. Benedict, in Proceedings of the Twelfth International Conference on High Magnetic Fields in the Physics of Semiconductors, edited by G. Landwehr and W. Ossau (World Scientific, Singapore, 1997), p. 95.

${ }^{12}$ K.A. Benedict and R.K. Hills, Phys. Rev. B 63, 235304 (2001).

${ }^{13}$ Yu.A. Bychkov, S.V. Iordanskii, and G.M. Eliashberg, Pis'ma Zh. Eksp. Teor. Fiz. 34, 496 (1981) [JETP Lett. 34, 473 (1981)].

${ }^{14}$ V.I. Fal'ko and S.V. Iordanskii, J. Phys.: Condens. Matter 4, 9201 (1992).

${ }^{15}$ S.M. Girvin, A.H. MacDonald, and P.M. Platzman, Phys. Rev. B 33, 2481 (1986).

${ }^{16}$ R.B. Laughlin, Phys. Rev. Lett. 50, 1395 (1983).

${ }^{17}$ R. E. Prange and S. M. Girvin, The Quantum Hall Effect (Springer, Heidelberg, 1987).

${ }^{18}$ T. Chakraborty and P. Pietilainen, The Fractional Quantum Hall Effect, 2nd ed. (Springer, New York, 1995).

${ }^{19}$ T. Ando, A.B. Fowler, and F. Stern, Rev. Mod. Phys. 54, 437 (1982).

${ }^{20}$ V. F. Gantmakher and Y. B. Levinson, Carrier Scattering in Metals and Semiconductors (North-Holland, Amsterdam, 1987). 